\title{
Tuberculosis vertebral con afectación pleural: reporte de un caso
}

\author{
Juan David Patiño Mateus* \\ María Alejandra Orejarena Alarcón** \\ Sergio Alberto Villabona Rosales*** \\ Andrés Felipe Roa Villabona****
}

\begin{abstract}
*Medicina XII semestre. Universidad Autónoma de Bucaramanga (UNAB). Grupo de Investigaciones Clínicas - Semillero de urgencias y trauma, Universidad Autónoma de Bucaramanga. Bucaramanga. Colombia.

**Medicina XI semestre. Universidad Autónoma de Bucaramanga (UNAB). Grupo de Investigaciones Clínicas - Semillero de urgencias y trauma, Universidad Autónoma de Bucaramanga. Bucaramanga. Colombia.

***Médico, Especialista en Urgenciología. Fundación Oftalmológica de Santander - FOSCAL. Docente asociado, Universidad Autónoma de Bucaramanga. Grupo de Investigaciones Clínicas - Semillero de urgencias y trauma, Universidad Autónoma de Bucaramanga. Bucaramanga. Colombia.

****Médico, Especialista en Epidemiología. Universidad Nacional de Santander - Universidad Autónoma de Bucaramanga. Grupo de Investigaciones Clínicas - Semillero de urgencias y trauma, Universidad Autónoma de Bucaramanga. Bucaramanga. Colombia.

Correspondencia: Dr. Juan David Patiño Mateus. Dirección: calle 21 \# 2- 61 Paseo del Puente. Conjunto residencial Paseo Real 1 Torre 12 Apto 147. Piedecuesta Santander. Correo: juanda18860@hotmail.com - jpatino120@unab.edu.co Teléfono: 3112847276
\end{abstract}

Resumen

La tuberculosis extrapulmonar representa hasta el $25 \%$ de todos los casos de tuberculosis. Los órganos más frecuentemente afectados son los ganglios linfáticos y la pleura. Alrededor del 10-11\% de casos de tuberculosis extrapulmonar tienen afectación osteoarticular y de ellos la mitad con compromiso vertebral. La infección es causada por la diseminación hematógena del bacilo desde un foco primario al hueso esponjoso de los cuerpos vertebrales torácicos o lumbares principalmente. El síntoma característico es el dolor crónico de la columna vertebral, generalmente en el sitio afectado, sin otros síntomas o signos concomitantes. La asociación de tuberculosis vertebral con afectación pleural sin un foco pulmonar establecido es infrecuente, se ha descrito en $2.5 \%$ de pacientes e intriga acerca de su fisiopatología. En Colombia, no se encontraron reportes similares. Se describe el caso de una paciente joven con historia de dolor lumbar crónico que debutó con síntomas respiratorios persistentes asociado a síntomas constitucionales; en quien se comprobó la infección por Mycobacterium tuberculosis en vértebras toracolumbares y pleura. Este caso evidencia una historia natural atípica de la enfermedad, en la cual el mecanismo fisiopatológico parece haber sido la diseminación directa por contigüidad, y resalta la importancia de la sospecha clínica para garantizar un diagnóstico y tratamiento oportuno. MÉD.UIS.2020;33(3): 67-73.

Palabras clave: Tuberculosis. Tuberculosis de la Columna Vertebral. Tuberculosis Pleural. Mycobacterium Tuberculosis. Enfermedad de Pott.

\section{Spinal tuberculosis with pleural involvement: a case report}

\begin{abstract}
Extrapulmonary tuberculosis represents up to $25 \%$ of all cases of tuberculosis. The most frequently affected organs are lymph nodes, pleura and bone. Around $10-11 \%$ of cases of extrapulmonary tuberculosis have osteoarticular involvement and a half of them present vertebral involvement. The infection is caused by hematogenous spread of the bacillus from a primary focus to the cancellous bone of the thoracic or lumbar vertebral bodies mainly. The characteristic symptom is the chronic pain in the spine, usually in the affected site, without other concomitant symptoms or signs. The association between vertebral tuberculosis and pleural involvement without an established pulmonary focus is infrequent, it has been described in $2.5 \%$ of patients, an intrigue about its pathophysiology. Similar cases in Colombia have not been reported. This article describes a case of a young patient with a history of chronic low back pain that debuts with persistent respiratory symptoms associated with constitutional symptoms; whose Mycobacterium tuberculosis infection was proven in thoracolumbar vertebrae and pleura. This case shows an atypical natural history of the disease, in which the pathophysiological mechanism of the disease would seem to have been the direct dissemination by contiguity, and emphasizes the importance of clinical suspicion to ensure timely diagnosis and treatment. MÉD.UIS.2020;33(3): 67-73.
\end{abstract}

Keywords: Tuberculosis. Tuberculosis. Spinal. Tuberculosis. Pleural Mycobacterium Tuberculosis. Pott’s Disease

Artículo recibido el 9 de agosto de 2020 y aceptado para publicación el 25 de octubre de 2020 . 
¿Cómo citar este artículo?:Patiño J, Orejarena M, Villabona S, Roa A. Tuberculosis vertebral con afectación pleural: Reporte de un caso. MÉD.UIS.2020;33(3):67-73. doi: 110.18273/revmed.v33n32020008

\section{Introducción}

La tuberculosis (TB) sigue siendo una causa importante de morbilidad y mortalidad a nivel mundial. En 2017, alrededor de 10 millones de personas presentaron TB y 1,3 millones murieron por esta causa'. Mientras que la región de las américas contribuyó sólo con el 3\% de la carga mundial de TB; específicamente en Sudamérica, la incidencia fue de 46,2 casos por cada 100.000 personas para $2017^{1}$. Según las estadísticas del Instituto Nacional de Salud (INS) de Colombia, durante el año 2018 se notificaron 13032 casos nuevos, con una tasa de incidencia de 26,9 casos por cada 100000 habitantes para todas las formas de TB y una mortalidad de 2 casos por cada 100000 habitantes².

La TB se clasifica en pulmonar y extrapulmonar. El sitio más común de infección es el pulmón’3 Sin embargo, el bacilo puede comprometer otros órganos por diseminación hematógena, linfática o incluso por contigüidad, pero con menor frecuencia3. A nivel mundial, la TB extrapulmonar representa $20-25 \%$ de todos los casos de tuberculosis4; los órganos más frecuentemente afectados son los ganglios linfáticos, la pleura y el hueso ${ }^{4}$. De los casos reportados en 2018 en Colombia, 17,3\% correspondieron a TB extrapulmonar; afectando principalmente la pleura $(42,9 \%)$, las meninges $(17,9 \%)$ y los ganglios linfáticos $(15,1 \%)^{2}$.

Los factores relacionados con el desarrollo de TB extrapulmonar incluyen la edad, el sexo femenino, la coinfección con el Virus de Inmunodeficiencia Humana (VIH) y comorbilidades como la enfermedad renal crónica, diabetes mellitus u otras causas de inmunosupresión (medicamentos inmunosupresores, cáncer, enfermedades autoinmunes, entre otras) $)^{4}$.

Aproximadamente, $10-11 \% 4,5$ de las formas extrapulmonares corresponden a TB osteoarticular, de los cuales hasta la mitad tienen tuberculosis vertebral o enfermedad de Pott ${ }^{5}$. El síntoma característico es el dolor de la columna vertebral crónico sin la compañía de otros síntomas o signos, lo que conlleva a un diagnóstico tardío, con deformidades de la columna vertebral y déficits neurológicos ${ }^{5}$.

La infección vertebral es causada por la diseminación hematógena del bacilo, vía arterial o venosa, desde un foco primario al hueso esponjoso de los cuerpos vertebrales ${ }^{5,6}$. Anatómicamente, el disco intervertebral es una estructura avascular, pero en la región subcondral de cada vértebra se forma un plexo arterial derivado de las arterias espinales anteriores y posteriores que facilita la infección en las regiones paradiscales. Por otro lado, el plexo venoso de Batson, que comunica las venas intercostales posteriores con el plexo vertebral, carece de válvulas y permite el flujo sanguíneo, y así mismo la diseminación, en ambas direcciones.

Con respecto a la TB pleural, esta puede ser secundaria a una infección primaria o a una reactivación de la enfermedad. En la mayoría de casos, su desarrollo parece estar relacionado con la ruptura de un foco caseoso pulmonar de localización subpleural hacia el espacio pleural7,8. Esto permite la entrada del Mycobacterium y de sus antígenos ocasionando una reacción de hipersensibilidad ${ }^{8}$. En aquellos pacientes en los que no se logra establecer un compromiso pulmonar, la afectación pleural puede ser el resultado de una reactivación o de una diseminación desde otro foco primario en la minoría de casos.

El curso clínico de la TB pleural puede ser agudo o subagudo; se caracteriza por fiebre, dolor torácico pleurítico y tos generalmente no productiva ${ }^{7,8}$. Algunos pacientes también pueden presentar disnea, diaforesis nocturna, pérdida de peso y fatiga ${ }^{7,8}$. La presentación simultánea de TB vertebral con TB pleural es infrecuente, se presenta en alrededor del $2.5 \%$ de pacientes ${ }^{9}$, e intriga acerca de su fisiopatología.

El objetivo de este artículo es presentar el caso de una paciente con tuberculosis en la que al parecer el foco primario de la enfermedad fue la infección vertebral con una posterior afectación pleural secundaria a una diseminación directa por contigüidad. Esto evidencia una historia natural atípica de la enfermedad. 


\section{Caso clínico}

Mujer de 34 años con antecedente de dolor en región dorso lumbar sin síntomas neurológicos de un año de evolución aproximadamente. Consultó por dolor pleurítico derecho de un mes de evolución, asociado a astenia y adinamia; en los últimos 15 días agregó tos seca, disnea de esfuerzo progresiva, diaforesis nocturna y vespertina con fiebre cuantificada en 39,6 ${ }^{\circ} \mathrm{C}$ y pérdida involuntaria de peso de $6 \mathrm{~kg}$. Al examen físico de ingreso estaba normotensa con taquicardia y taquipnea, febril, sin desaturación y a la auscultación pulmonar con disminución del murmullo vesicular de predominio derecho. En el laboratorio presentaba anemia normocítica normocrómica y linfopenia moderada con elevación de reactantes de fase aguda (Ver tabla 1).

Inicialmente se realizó una Tomografía Computarizada (TAC) de tórax en donde se observó un derrame pleural derecho escaso conformando imagen de pseudo tumor evanescente con engrosamiento pleural hacia la base pulmonaripsilateral y destrucción ósea que comprometía cuerpos vertebrales de T12 y
Tuberculosis vertebral con afectación pleural: reporte de un caso

L1 y sugieren realizar estudios complementarios. Se realizó una Resonancia Magnética Nuclear (RMN) de columna dorsolumbar con hallazgos sugestivos de espondilodiscitis de T12 y L1 con gran compromiso de cuerpos vertebrales y herniación de disco anterior (Ver Figura 1).

Por la severidad del cuadro clínico y la respuesta inflamatoria sistémica, se pensó en infección piógena en columna dorsal y tejidos blandos, y se inició tratamiento antibiótico de amplio espectro con vancomicina y meropenem; sin embargo, la paciente continuó con fiebre y deterioro progresivo del estado general. Con una alta sospecha de tuberculosis, dada la evolución clínica y el compromiso respiratorio y vertebral, se retiró el manejo instaurado y se inició tratamiento tetraconjugado con isoniazida, rifampicina, etambutol y pirazinamida de forma empírica. La prueba de VIH (técnica ELISA), los hemocultivos periféricos, las baciloscopias seriadas de esputo y el cultivo para gérmenes comunes resultaron negativos. El test de Mantoux (o prueba cutánea de la tuberculina) mostró una induración de $6 \mathrm{~mm}$, reacción positiva según valores de referencia del laboratorio.

Tabla 1. Cronología de los análisis de laboratorio.

\begin{tabular}{|c|c|c|c|c|c|c|c|}
\hline VR & $\begin{array}{c}\text { Hb. } \\
\mathbf{1 2 . 3} \mathbf{- 1 5 . 3}\end{array}$ & $\begin{array}{c}\text { Hemat. } \\
\mathbf{4 1 0 0 - 5 1 0 0}\end{array}$ & $\begin{array}{c}\text { Leuco. } \\
\mathbf{4 . 4 - 1 1 . 3}\end{array}$ & $\begin{array}{c}\text { Neut. } \\
\mathbf{2 - 7}\end{array}$ & $\begin{array}{c}\text { Linf. } \\
\mathbf{1 5 0 0}-\mathbf{4 0 0 0}\end{array}$ & $\begin{array}{c}\text { Pqtas. } \\
\mathbf{1 5 0}-\mathbf{4 4 0}\end{array}$ & $\begin{array}{c}\text { PCR } \\
\mathbf{0 , 6}-\mathbf{5 , 0}\end{array}$ \\
\hline Día 0 & 10 & 3460 & 5.2 & 3.7 & 870 & 423 & 302,57 \\
\hline Sem. 1 & 8,6 & 3111 & 3.9 & 2.53 & 730 & 304 & - \\
\hline Sem. 2 & 6,7 & 3240 & 4.1 & 2.72 & 820 & 296 & - \\
\hline Sem.3 & 9,5 & 3360 & 3.6 & 2.28 & 810 & 225 & - \\
\hline Sem.4 & 9,3 & 3250 & 2.89 & 1.64 & 620 & 232 & - \\
\hline Sem.5 & 10,4 & 3610 & 3.64 & 1.97 & 870 & 272 & - \\
\hline Sem.6 & 10,6 & 3730 & 4.23 & 2.67 & 780 & 288 & - \\
\hline Sem.8 & 10,8 & 3730 & 3.28 & 1.80 & 800 & 211 & - \\
\hline Sem.10 & 8,4 & 2890 & 7.94 & 6.53 & 480 & 127 & 15,14 \\
\hline Sem.11 & 9,2 & 3190 & 8.81 & 6.87 & 704 & 207 & 215,35 \\
\hline
\end{tabular}

VR: Valores Referencia; Sem: semana; ALT: Alanin aminotransferasa (U/L); AST: Aspartato aminotransferasa (U/L); Hemat: Hematíes x103/ mm3; Hb: Hemoglobina (gr/dL); Leuco: Leucocitos/mm3; Linf: Linfocitos x103/uL; Neu: Neutrófilos x103/uL; PCR: Proteína C reactiva (mg/ dL); Pqtas: Plaquetas x103/mm3

Fuente: autores.

Se realizó decorticación pulmonar con pleurectomía parcial derecha, evidenciándose paquipleuritis severa y adherencias pleurales con estudio histopatológico Ziehl Neelsen positivo. Con el fin de confirmar el agente etiológico de la enfermedad vertebral y con la disponibilidad del servicio de radiología intervencionista, se tomó biopsia vertebral guiada por TAC con cultivo para gérmenes comunes, baciloscopia y PCR para Mycobacterium tuberculosis inicialmente negativos. Dada la linfopenia persistente, se hizo cuantificación de linfocitos (CD3: 489,3; CD4-H: 262,7 y CD8: 225,7) con una segunda 
prueba de VIH (técnica ELISA) y virus linfotrópico de células T humano tipo I (HTLV-I) no reactivas. Se realizaron estudios de autoinmunidad con pruebas de complemento, ANAS y factor reumatoide negativos. No se cuenta con analíticas previas para determinar estado basal y tendencia de las líneas celulares. El reporte final del cultivo de esputo fue negativo para bacilos ácido alcohol resistentes (BAAR) a las 8 semanas de incubación.

En vista de la inestabilidad mecánica de la columna, se realizó corpectomía anterior de T12 y L1 más discectomía con colocación de sistema de artrodesis anterior de columna e injerto óseo con duroplastia y reducción de fractura. En los hallazgos macroscópicos del procedimiento, se encontró abundante material caseoso y granulomatoso a nivel óseo y en tejidos blandos perivertebrales desde T11 hasta L2, con masa de igual contenido dentro del canal espinal que comprimía el cono medular. Una semana después del primer abordaje quirúrgico, se llevó a cabo una descompresión medular posterior con artrodesis transpedicular.

El reporte final del cultivo para micobacterias en medio líquido de biopsia vertebral fue positivo para BAAR, con prueba rápida de identificación para el complejo Mycobacterium (GeneXpert) positivo para Mycobacterium tuberculosis y estudio molecular de susceptibilidad a antituberculosos con sensibilidad a isoniacida y rifampicina.
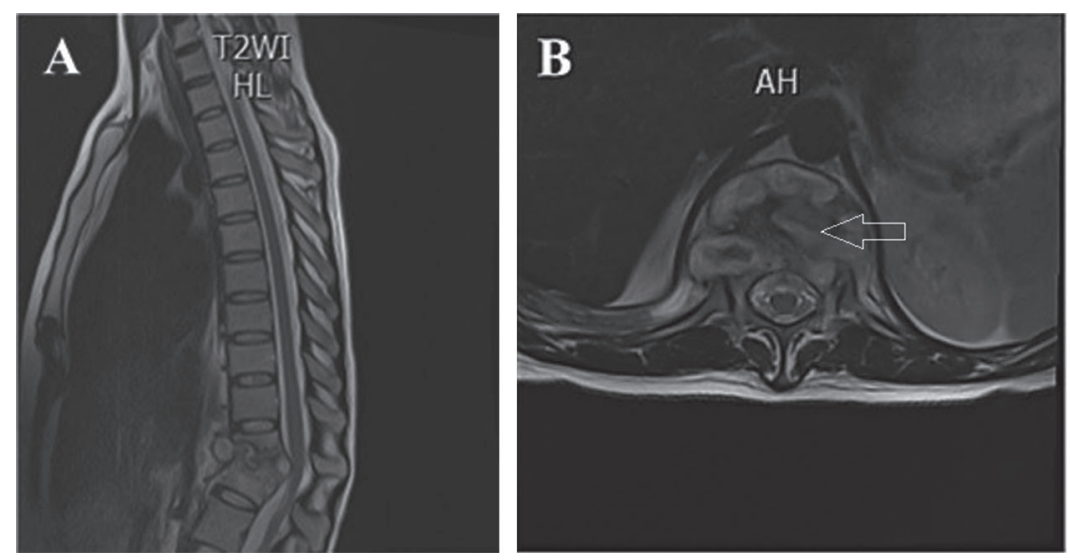

Figura 1. RNM de columna dorsolumbar. Corte sagital (A): fractura patológica con disminución de un $90 \%$ de la altura del cuerpo vertebral de T12 y del $10 \%$ del cuerpo vertebral de L1 con pérdida de la morfología del disco intervertebral. Corte axial (B): material hiperintenso a nivel de disco intervertebral T12-L1 y colección en el espacio epidural anterior y a nivel de los tejidos blandos para y prevertebrales Fuente: autores.

Durante la hospitalización, la paciente presentó elevación en los niveles de ALT aparentemente como efecto secundario de la pirazinamida, por lo que se suspendió el tratamiento antituberculoso de forma temporal. El reinicio se hizo de forma escalonada, inicialmente con etambutol y moxifloxacina, y posteriormente se incorporó rifampicina e Isoniazida con adecuada evolución clínica. La paciente estuvo hospitalizada durante 14 semanas y fue dada de alta con seguimiento ambulatorio por infectología, neumología y clínica del dolor.

\section{Discusión}

La presentación clínica de la TB extrapulmonar es inespecífica y puede simular otras enfermedades según el sitio afectado. Webster y Shandera identificaron que el género femenino y la edad menor de 45 años son factores de riesgo asociados a TB extrapulmonar ${ }^{10}$. Un estudio de cohorte retrospectiva en China evidenció que las personas entre 25- 44 años desarrollaron más TB extrapulmonar comparado con los mayores de 65 años, principalmente TB linfática, meníngea y pleural. En ese mismo estudio, las mujeres tuvieron mayor riesgo de desarrollar formas extrapulmonares, con mayor probabilidad de TB esquelética, diseminada, linfática y genitourinaria ${ }^{11}$. Otros factores de riesgo descritos son el uso de corticoides o tener cáncer $^{12}$, en cuanto que afectan el estado inmunológico. Esto sugiere que la paciente del caso clínico pudiese tener cierto perfil de riesgo, aunque en ella se descartó la infección por VIH y no se documentaron otras patologías que produjeran algún tipo de inmunodeficiencia. Una limitante de este caso es no contar con una analítica previa que permitiera determinar si la paciente tenía una 
Septiembre - diciembre

inmunodeficiencia preexistente, dada la linfopenia que presentaba en el momento de la admisión y, de ser así, hubiese sido un factor predisponente de primer orden para el desarrollo de la infección tuberculosa.

La TB osteoarticular representó $4,9 \%$ de los casos de TB extrapulmonar en Colombia en 2018², en contraste con los reportes de otros países en los que se describe como un sitio de afectación frecuente ${ }^{4,5}$. La localización más común de infección es la columna vertebral hasta en la mitad de los $\operatorname{casos}^{5}$, seguido de otras articulaciones como la rodilla, la cadera y el hombro ${ }^{12}$.

La columna torácica baja y lumbar son las estructuras más frecuentemente afectadas ${ }^{5}$. Dharmalingam observó un compromiso de las vértebras torácicas y lumbares en $30,3 \%$ y $27,2 \%$, respectivamente ${ }^{13}$. Sin embargo, en otro estudio el compromiso fue principalmente lumbar (55\%), torácico (45\%) y menos frecuente toracolumbar $(10 \%)^{13}$. En el caso clínico presentado, hubo afectación toracolumbar, aunque principalmente torácica y en menor proporción lumbar.

La progresión de la tuberculosis vertebral es insidiosa, los pacientes consultan tardíamente cuando el dolor se hace incapacitante, hay deformidad evidente o síntomas neurológicos ${ }^{14}$. Es por ello que se requiere un alto índice de sospecha para realizar una detección temprana. El dolor de la columna vertebral crónico es el síntoma más frecuente; este puede ser fluctuante e inespecífico, pero generalmente se localiza en el segmento espinal afectado ${ }^{12}$, con una evolución promedio de 4 a 11 meses $^{14}$. El dolor es el único síntoma hasta en $61 \%$ de los casos y $20-30 \%$ presentan síntomas constitucionales como pérdida de peso, diaforesis y fiebre nocturna, indicativos de enfermedad activa ${ }^{14}$. La paciente refirió dolor lumbar como único síntoma por aproximadamente un año, el cual no fue estudiado adecuadamente y sólo tomó importancia cuando debutó con los síntomas respiratorios.

El diagnóstico de TB vertebral se basa en la correlación de los hallazgos clínicos e imagenológicos. La RMN es la imagen de elección con una sensibilidad del $96 \%$ y una especificidad del $92 \% 15$; esta prueba permite demostrar el compromiso de los cuerpos vertebrales, destrucción del disco, absceso frío, colapso vertebral y deformidades espinales ${ }^{11}$. La confirmación etiológica se realiza con estudios histopatológicos
Tuberculosis vertebral con afectación pleural: reporte de un caso

de una muestra obtenida, generalmente, por biopsia guiada por TAC. La baciloscopia y el cultivo son positivos para BAAR hasta en $52 \%$ y $83 \%$ de las biopsias, respectivamente ${ }^{16}$. En muestras paucibacilares en las que la baciloscopia es negativa y el cultivo positivo, como en el caso presentado, el uso de pruebas rápidas como Gene Xpert MTB/RIF son de gran utilidad en la detección del complejo Mycobacterium tuberculosis. Wen et al. evidenciaron que el Xpert MTB/RIF tiene una sensibilidad del $81 \%$ y una especificidad del $83 \%$ en la detección de TB musculoesquelética ${ }^{17}$; sin embargo, hubo una heterogeneidad moderada a alta en los estudios incluidos en el meta-análisis.

La presencia de tuberculosis pulmonar en pacientes con compromiso vertebral es frecuente. En un estudio retrospectivo, $67 \%$ de pacientes con TB vertebral tenían radiografía de tórax sugestiva de TB pulmonar'13. No obstante, en los estudios imagenológicos de la paciente no se evidenciaron lesiones que comprometan el parénquima pulmonar; adicionalmente, las baciloscopias seriadas de esputo y el cultivo del mismo para micobacterias fueron negativos.

La temporalidad y evolución de los síntomas del caso presentado sugieren que la afectación pleural que se evidenciaba en la paciente podría ser secundaria a la diseminación desde la colección para y prevertebral por un mecanismo de contigüidad tisular. Anatómicamente, la fascia endotorácica separa la pleura parietal de la cavidad torácica y cubre los músculos intercostales y las costillas, hacia atrás se continúa con la fascia perivertebral que cubre los cuerpos vertebrales y los discos. Por lo tanto, la fascia endotorácica parece ser la única estructura que tiene una continuidad anatómica con la fascia perivertebral.

En 1955 en Inglaterra, Stevenson reunió información de 23 casos de derrames pleurales que ocurrieron después del inicio de los síntomas de una espondilodiscitis tuberculosa torácica o toracolumbar, en ausencia de compromiso pulmonar. La mayor parte de las lesiones vertebrales se ubicaron en la región torácica, desde T6 a T12, y en las primeras vértebras lumbares ${ }^{18}$; el derrame pleural se detectó de 2 meses a 7 años después del inicio de los síntomas osteoarticulares y no se documentó una razón diferente a la infección vertebral ${ }^{18}$. En otro reporte, se documentaron 2 casos con extensión lateral de un absceso frío con afectación de la pleura parietal, sin evidencia de enfermedad pulmonar ${ }^{19}$. 
En la paciente del caso presentado, la vértebra más afectada fue T12 con cierto compromiso de L1 y el derrame pleural parece haber aparecido después de un año de cursar con síntomas sugestivos de TB vertebral.

Le Guillou et al. reportaron el caso de una joven de 20 años con TB vertebral y engrosamiento pleural; quien al igual que la paciente, no tenía antecedente de tabaquismo, tenía prueba de VIH negativa, cursó con un cuadro clínico respiratorio de corta evolución, con baciloscopias de esputo negativas, compromiso de las vértebras torácicas y colecciones epidurales y paravertebrales ${ }^{20}$. A diferencia del caso presentado, esta joven tenía una afectación pleural bilateral y no había evidencia de BAAR en la biopsia pleural.

Teniendo en cuenta lo anterior, cuando el derrame pleural es posterior al inicio de la enfermedad vertebral, en ausencia de enfermedad pulmonar y descartando otras posibles etiologías, es plausible considerar que su causa sea una propagación directa de la infección tuberculosa desde el foco vertebral y paravertebral.

\section{Conclusión}

Este es el primer caso reportado en Colombia y refuerza la hipótesis fisiopatológica de infección pleural por contigüidad en contexto de una TB vertebral establecida. Adicionalmente, ilustra la importancia de considerar la TB vertebral entre los diagnósticos diferenciales del dolor dorsolumbar crónico, incluso en personas previamente sanas y sin inmunosupresión documentada. La sospecha clínica con el apoyo de pruebas de imagen permitirá hacer un diagnóstico y tratamiento oportuno, evitando así complicaciones que prolongan la estancia hospitalaria y afectan negativamente la calidad de vida.

\section{Consideraciones éticas}

Existe consentimiento informado firmado por la paciente para la publicación de este reporte de caso y de las imágenes.

\section{Conflicto de intereses}

Ninguno

Financiación

Ninguna.

\section{Agradecimientos}

Al Dr. Paul Anthony Camacho López - Médico epidemiólogo y al Dr. Héctor Julio Amaya Santiago Médico infectólogo, por su apoyo y acompañamiento permanente en la elaboración del artículo.

\section{Referencias bibliográficas}

1. Woodman M, Haeusler IL, Grandjean L. Tuberculosis Genetic Epidemiology: A Latin American Perspective. Genes. 2019; 10(1):53.

2. López MP. Informe de evento Tuberculosis, Colombia, 2018 [Internet]. Bogotá: Instituto Nacional de Salud; 2019. Disponible en: https:/www.ins.gov.co/buscador-eventos/Informesdeevento/ TUBERCULOSIS_2018.pdf

3. Pezzella AT. History of Pulmonary Tuberculosis. Thorac Surg Clin. 2019;29(1):1-17.

4. Ramírez-Lapausa M, Menéndez-Saldaña A, Noguerado-Asensio A. Extrapulmonary tuberculosis: an overview. Rev Esp Sanid Penit. 2015;17(1):3-11.

5. Ali A, Musbahi O, White VLC, Montgomery AS. Spinal Tuberculosis: A Literature Review. JBJS Rev. 2019;7(1):e9.

6. Rajasekaran S, Soundararajan DCR, Shetty AP, Kanna RM. Spinal Tuberculosis: Current Concepts. Global Spine J. 2018;8(4 Suppl):96S-108S.

7. Shaw JA, Diacon AH, Koegelenberg CFN. Tuberculous pleural effusion. Respirology. 2019;24(10):962-71.

8. Cohen LA, Light RW. Tuberculous Pleural Effusion. Turk Thorac J. 2015;16(1):1-9.

9. Batirel A, Erdem H, Sengoz G, Pehlivanoglu F,Ramosaco E, Gülsün S, et al. The course of spinal tuberculosis (Pott disease): results of the multinational, multicentre Backbone-2 study. Clin Microbiol Infect. 2015;21(11):1008.e9-1008.e18.

10. Webster AS, Shandera WX. The extrapulmonary dissemination of tuberculosis: A meta-analysis. Int J Mycobacteriol. 2014;3(1):9-16.

11. Pang Y, An J, Shu W, Huo F, Chu N, Gao M, et al. Epidemiology of Extrapulmonary Tuberculosis among Inpatients, China, 20082017. Emerg Infect Dis. 2019;25(3):457-464.

12. Peirse M, Houston A. Extrapulmonary tuberculosis. Med. 2017;45(12):747-752.

13. Dharmalingam M. Tuberculosis of the spine the Sabah experience. Epidemiology, treatment and results. Tuberculosis (Edinb). 2004;84(1):24-28.

14. Garg RK, Somvanshi DS. Spinal tuberculosis: a review. J Spinal Cord Med. 2011; 34 (5):440-454.

15. Maftah M, Lmejjati M, Mansouri A, El Ebbadi N, Bellakhdar F. Mal de Pott a propos de 320 cas. Médecine du Maghreb. 2001; No. 90.

16. Francis I, Das D, Luthra U, Sheikh Z, Sheikh M, Bashier M. Value of radiologically guided fine needle aspiration cytology (FNAC) 
Septiembre - diciembre

in the diagnosis of spinal tuberculosis: a study of 29 cases. Cytopathology. 1999;10 (6):390-401.

17. Wen H, Li P, Ma H, Lv G. Diagnostic accuracy of Xpert MTB/RIF assay for musculoskeletal tuberculosis: a meta-analysis. Infect Drug Resist. 2017;10: 299-305.

18. Stevenson FH. The natural history of pleural effusion and orthopaedic tuberculosis. J Bone Joint Surg Br. 1955; 37-B (1):80-91.
Tuberculosis vertebral con afectación pleural: reporte de un caso

19. Malhotra HS, Garg RK, Raut TP. Pleural involvement in spinal tuberculosis. Am J Trop Med Hyg. 2012;86(4):560. PMID: 22492135.

20. Le Guillou F, Hubscher P, Cuvelier A, Quieffin J, Guyonnaud C-D, El Haite A, et al. Bilateral pleural thickening pseudo-tumour due to tuberculosis. Rev Mal Respir. 2002;19(4):515-7. PMID: 12417867 